\title{
Influence of attended repetition trials on negative priming in younger and older adults
}

\author{
PATRICIA M. SIMONE, KAREN AHRENS, \\ KARIN ELAINE GOODSON FOERDE, and MICHAEL SPINETTA \\ Santa Clara University, Santa Clara, California
}

\begin{abstract}
A lengthened response time when a distractor becomes a target, called negative priming, is an undisputed phenomenon in selective attention, yet just what the underlying mechanism responsible for negative priming is has not been resolved. In this study, the proportion of attended repetition trials was manipulated in order to test the predictions of three theories that have been proposed for explaining spatial negative priming: distractor suppression (e.g., Tipper, 1985), episodic memory retrieval (e.g., Neill, Valdes, \& Terry, 1995), and novelty bias (e.g., Milliken, Tipper, Houghton, \& Lupiáñez, 2000). The results supported the proposal that a novelty bias, which is flexible and can be overridden, is the primary mechanism responsible for priming in spatial tasks. Memory retrieval obscured the novelty bias for target processing, was more selective in older adults, and did not affect distractor processing. Novelty bias and distractor suppression may share the same inhibitory attentional mechanism.
\end{abstract}

Negative priming, the slowed responding to a target that has recently appeared as a distractor, has been a major focus of study in selective attention since Tipper (1985), who presented young adults with overlapping target and distractor line drawings. On prime trials, the target drawing to be named (e.g., a kite) was outlined in red, and the distractor drawing (e.g., a trumpet) was outlined in green. On the subsequent probe trials, the prime distractor (i.e., the trumpet) was outlined in red and required a response. As compared with a control condition, in which the stimuli in the probe were unrelated to those in the prime, the participants were slower to respond to the target in the ignored repetition probe trial. This increase in response time has been referred to as negative priming, and its presence suggests some sort of processing of the prime trial distractor.

\section{Accounts of Negative Priming: Distractor Suppression and Memory Retrieval}

One of the first theories proposed to explain negative priming was that of distractor suppression, or inhibition of the distractor in the prime trial (Tipper, 1985). The extra time required to overcome this sustained suppression in the probe trial results in a slowdown in responding. A second theory argues that negative priming results from memory retrieval of the prime distractor during the probe trial (Neill, Valdes, Terry, \& Gorfein, 1992; see also Park

This research was supported by internal grants and a sabbatical awarded by Santa Clara University to P. M. S. We are thankful to Cris Rys for the computer program, Megan Cristina and Joe Manns for collection of pilot data, and Robert Numan for comments on earlier drafts. We also thank Michael Kane, Bruce Milliken, and an anonymous reviewer for suggestions. Correspondence regarding this article should be addressed to P. M. Simone, Department of Psychology, Santa Clara University, Santa Clara, CA 95053 (e-mail: psimone@scu.edu).
\& Kanwisher, 1994, for a related view). For example, in Tipper's study, repeating the picture of the trumpet (used as the distractor in the prime trial) as the target in the probe trial evoked the memory of the trumpet from the prime trial. However, because the trumpet was the distractor in the prime trial, a conflict between the memory of the previous trial and the current trial ensued. Memory retrieval theory states that negative priming results from the increased time required to resolve this conflict and to make the proper selection in the probe trial.

In a review of the negative priming literature, May, Kane, and Hasher (1995; see Fox, 1995, and Neill, Valdes, \& Terry, 1995, for additional reviews) argued that the experimental design may determine whether memory retrieval or distractor suppression is the operative mechanism in any given negative priming task. May et al. suggested that the inclusion of attended repetition trials may induce memory retrieval in both attended and ignored repetition trials. In attended repetition trials, the prime target is presented again as the probe target, and whereas responding is slower and/or less accurate in ignored repetition trials (negative priming), responding is faster and/ or more accurate on attended repetition trials (repetition priming), as compared with control trials. This benefit in responding due to repetition of the target is presumed to occur because of the retrieval of the memory of the target from the previous trial.

\section{Negative Priming Research in Older Adults}

Negative priming and aging research has been plagued with inconsistent results, with older adults sometimes showing negative priming (e.g., Schooler, Neumann, Caplan, \& Roberts, 1997; Simone \& Baylis, 1997; Simone \& McCormick, 1999; Sullivan \& Faust, 1993; Sullivan, Faust, \& Balota, 1995), and sometimes not (e.g., Connelly \& Hasher, 1993; McDowd \& Oseas-Kreger, 1991). In an 
effort to resolve this conflict, some research has suggested that the difference lies in whether the negative priming results from memory retrieval or distractor suppression and that older adults are able to engage memory retrieval processes, but not distractor suppression (Kane, May, Hasher, Rahhal, \& Stoltzfus, 1997).

Kane et al. (1997) performed a series of experiments in which the question of which mechanism is responsible for negative priming in younger and older adults was investigated. They found that older adults exhibited negative priming only under conditions designed to elicit memory retrieval, such as the inclusion of repeated target trials (attended repetition). In the experiment that contained attended repetition trials, they found that the older adults exhibited negative priming only when they also exhibited a slowdown in the target-to-distractor condition, as compared with control trials. In agreement with the proposal of May et al. (1995), they argued that a slowdown in this target-to-distractor condition indicates the use of memory retrieval in the task. In another study, Kane, Hasher, Stoltzfus, Zacks, and Connelly (1994) failed to find negative priming in older adults. Correspondingly, in this study, older adults did not show a slowdown on target-to-distractor trials. The primary difference between the two studies (Kane et al., 1994; Kane et al., 1997) was the inclusion of attended repetition trials to encourage memory retrieval in Kane et al.'s (1997) study.

Two explanations have been proposed to account for the contradictory findings regarding negative priming in older adults. The first theory, examined above, emphasizes the mechanisms underlying negative priming - specifically, memory retrieval and distractor suppression. An alternative account points to methodological differences in experimental procedure. For example, Kane and colleagues examined negative priming by using nonspatial, identitybased tasks, in which the response was to voice the target name. In alternative negative priming procedures, spatial, location-based tasks are used, in which the response is to manually select the target on the basis of its location. These procedural differences may affect factors that influence negative priming (Connelly \& Hasher, 1993; May et al., 1995). In fact, several studies with identity-based tasks have reported no negative priming in older adults (e.g., Connelly \& Hasher, 1993; McDowd \& Oseas-Kreger, 1991), and several location-based studies have reported significant negative priming in this age group (e.g., Connelly \& Hasher, 1993; Simone \& Baylis, 1997; Simone \& McCormick, 1999).

Some researchers have speculated that suppression of distractor identity may be compromised in aging (Connelly \& Hasher, 1993), meaning that, in older adults, any negative priming that is observed results from memory retrieval (Kane et al., 1997). These results appear to account for the inconsistent findings of negative priming in older adults: Negative priming in older adults can result from memory retrieval, but not from suppression of distractor identity. However, some studies have indicated that there is preserved identity negative priming in older adults that results from distractor suppression (Sullivan \& Faust,
1993; Sullivan et al., 1995). Interestingly, identity negative priming has been found in older adults even in experiments designed to decrease the influence of memory retrieval-for example, by not including attended repetition trials (Gamboz, Russo, \& Fox, 2000; Schooler et al., 1997). Negative priming in older adults appears to be different from the negative priming found in younger adults. Why older adults sometimes show negative priming and sometimes do not has not yet been resolved.

Kane and colleagues (Kane et al., 1994; Kane et al., 1997) manipulated the influence of memory retrieval on negative priming in identity-based tasks and found that encouraging memory retrieval of the target (in attended repetition trials) had a carryover effect on distractor processing, causing negative priming in older adults that was attributed to memory retrieval. Since the procedural differences between spatial and identity tasks may influence negative priming and older adults typically show negative priming in spatial tasks, it is relevant to examine the relative contributions of memory retrieval of target information on negative priming in spatial localization tasks in younger and older adults.

In addition to memory retrieval and distractor suppression theories, an additional account of negative priming must be addressed when negative priming in a spatial localization task is examined. Recent results (Buckolz, Boulougouris, O’Donnell, \& Pratt, 2002; Christie \& Klein, 2001; Milliken, Tipper, Houghton, \& Lupiáñez, 2000) suggest that the negative priming observed in spatial localization tasks may result from an attention bias to novelty, an effect called inhibition of return (Posner \& Cohen, 1984). The slowdown when a distractor was repeated as a target could then be seen as a bias against returning attention to a location that had previously been inspected.

In Milliken et al.'s (2000) study, the participants were presented with two Xs in different colors, located in two of four possible locations on the computer screen. A central color cue that matched one $\mathrm{X}$ indicated which $\mathrm{X}$ was the target and was presented simultaneously with the two Xs. The participants made a joystick movement (up, down, left, or right) indicating the location of the target that matched the color of the central cue. Milliken et al. found that the participants were slower in all conditions in which a location was repeated between trials, whether that location had contained a target (as in attended repetition trials) or a distractor (as in ignored repetition trials). Since attended repetition trials typically result in faster responding times (repetition priming), Milliken et al. concluded that the slowdown in responding in the attended repetition trials was due to an attentional bias to examine novel locations. It may be that negative priming and inhibition of return rely on the same attentional mechanism (Buckolz et al., 2002; Milliken et al., 2000). Or, as Christie and Klein (2001) suggested, any slowdown observed in older adults when a distractor becomes a target is the result of inhibition of return, rather than of negative priming.

In sum, there are conflicting findings regarding negative priming in older adults, with some studies showing negative priming (Sullivan \& Faust, 1993) and others not 
(McDowd \& Oseas-Kreger, 1991). Current theories suggest that negative priming may result from suppression of the distractor (Tipper, 1985), memory retrieval conflict (Neill et al., 1992), or an attentional bias to detect novelty (Milliken et al., 2000). It has been suggested that older adults are unable to suppress distractors and, therefore, that any negative priming observed in this population must be the result of another mechanism, such as memory retrieval (Kane et al., 1997) or novelty bias (Christie \& Klein, 2001). If experimental design can influence whether memory retrieval or distractor suppression is responsible for negative priming in identity-based tasks (May et al., 1995), it is important to determine whether design manipulations also influence location-based tasks.

Kane et al. (1997) suggested that inclusion in an experiment of trial pairs in which the prime target is repeated as the probe distractor (target to distractor) might reveal the mechanism that accounts for any negative priming in that experiment. They suggested that a slowdown in the targetto-distractor condition, as compared with a control trial, would indicate that the experiment induces memory retrieval, because the repetition of the target as the distractor creates a memorial conflict. On the other hand, they suggested that no slowdown in the target-to-distractor condition, as compared with a control trial, would reveal that the experiment does not induce retrieval, so that any evident negative priming most likely results from distractor suppression. Although the distractor suppression theory of negative priming does not make specific predictions about the outcome of this probe trial condition, a novelty bias account predicts a target-to-distractor facilitation, due to a bias to avoid a location that was recently attended to. Recall that Kane and colleagues (Kane et al., 1994; Kane et al., 1997) included this target-to-distractor condition and found that older adults showed negative priming only when they also were slower on target-to-distractor trials in an identity negative priming task.

In the present study, we examined the influence of variable proportions $(0 \%, 25 \%$, and $50 \%)$ of attended repetition trials on three probe trial types: attended repetition (the target is repeated as a target), target-to-distractor trials (the target is repeated as a distractor), and ignored repetition (the distractor is repeated as a target). The question was whether increasing the proportion of attended repetition trials would result in a greater influence of memory retrieval of target and distractor information, thereby affecting repetition priming (faster responses when the target is repeated as a target), target-to-distractor responding (slowdown due to memory conflict between prime and probe trials), and negative priming (slower responses when the distractor becomes a target), as was found by Kane et al. (1997) in an identity-based negative priming task.

The distractor suppression theory of negative priming assumes that responding to a distractor that was recently a target will be slowed because of residual inhibition or suppression associated with the stimulus. If negative priming results from distractor suppression, and not from memory retrieval, attended repetition trials in any amount
$(0 \%, 25 \%$, or $50 \%)$ should not have an effect on negative priming. The distractor suppression theory does not make specific predictions about the effect of repeated targets on repetition priming or target-to-distractor responding, since both of these conditions involve target processing, not distractor processing.

Lastly, if priming results from an attentional bias to detect novelty and attention is inhibited from returning to a previously attended location, participants should be slower to select the target when the target is repeated as a target (attended repetition) and when the distractor is repeated as the target (ignored repetition), because the current target is in a previously attended location and the bias is to avoid previously attended locations. Similarly, participants should be faster when the distractor becomes the target (target to distractor), because the current distractor location was previously attended to and, therefore, attention is less likely to return to this location.

Responses were slower when location was repeated in the Milliken et al. (2000) task, regardless of whether the trial was attended or ignored repetition. However, Milliken et al. did find repetition priming, or faster responses to target repetition trials than to control trials, when the location and the color of the target stimulus were the same between trials. They speculated that the tendency to detect novel events may be overridden or obscured by a separate mechanism that favors repeated events. Increasing the saliency between trials, such as adding color or identity to location repetition, increases the efficiency of the second mechanism and increases the probability that the novelty bias will be overridden.

The procedure in the present experiment should result in the overriding of the novelty bias in target processing, resulting in faster responding to repeating targets than in nonrepeating target trials. In this task, location, color, and identity are repeated across trials for all probe conditions. A probe target may be repeated as a target stimulus in the same location and with the same identity and color as the prime trial target (attended repetition), or a probe target may be repeated as a distractor in the same location and with the same identity and color (target to distractor). This feature repetition, along with location repetition, should facilitate the obscuring of the bias to avoid the repeated location. In addition, the presence of many repeating targets $(25 \%$ or $50 \%)$ may override the novelty bias.

If the novelty bias is overridden for target processing and if repetition priming is found in this spatial localization task, responding on target-to-distractor and ignored repetition trials may also be influenced, as was the case in Kane et al.'s (1997) identity-based task. If participants are faster on trials in which a target is repeated because their novelty bias mechanism is obscured, there may be carryover effects that can be measured in the target-todistractor trials. In particular, if participants are aware of the benefit of returning to the location of the previous target, they may abandon the novelty bias entirely and search the location of the previous target first, if it contains a stimulus. Target selection will be faster on attended repetition trials and slower when the target becomes a distractor. 
Kane et al. (1997) found negative priming in older adults only with the addition of attended repetition in their identity-based task. Their findings suggest that manipulations of target processing can influence distractor processing. Specifically, memory retrieval of target repetition made it more likely to retrieve the memory of distractor information, leading to negative priming. Whether this carryover effect from target to distractor processing occurs in location-based tasks has not been determined.

Milliken et al. (2000) showed that the novelty bias could be overridden for target processing, but not for distractor processing. Although Milliken et al.'s results suggest that the bias to detect novel events may be overridden when there is an advantage in returning to a previously attended location, they did not find a similar facilitation in response times (RTs) when both the location and the color of the distractor were repeated as the target (ignored repetition trial). Rather, they continued to find longer RTs, or negative priming. This finding suggests that different mechanisms may be responsible for target and distractor processing in spatial localization tasks. The novelty bias may be overridden for target processing, but it is still responsible for processing of the distractor. However, Milliken et al. did not include variable proportions of attended repetition trials. It may be possible to override the novelty bias in distractor processing by increasing the probability that targets will be repeated.

Manipulating the memory retrieval contribution in this selective attention task may affect the responding of older adults differently than that of younger adults. If negative priming in older adults is due to memory retrieval (Kane et al., 1997), older adults will be expected to show negative priming only when other conditions implicate memory retrieval as the mechanism-for example, repetition priming and a slowdown in target-to-distractor responding. If negative priming in spatial localization tasks can be explained by a novelty bias (Christie \& Klein, 2001), the same patterns of results will be expected for younger and older adults, since it has been demonstrated that there is no effect of age on location-based inhibition of return (McCrae \& Abrams, 2001). If negative priming is due to distractor suppression, target processing may not influence distractor processing, and an effect of attended repetition trials on target processing should not have any impact on negative priming.

\section{METHOD}

\section{Participants}

A total of 74 undergraduates ( 21 of them male and 53 female) were recruited from the participant pool at Santa Clara University and received course credit for their participation. Their ages ranged from 18 to 27 years, with an average age of 18.75 years $(S D=1.3)$. The average education for these young participants was 13 years, with a range of 13-16 years. A total of 66 older adults ( 25 of them male and 41 female) were recruited from a senior center in the community surrounding Santa Clara University. Their ages ranged from 52 to 91 years, with an average of 71.5 years $(S D=8.1)$. The average educational level of the older participants was 13.5 years, with a range of 8-20 years. The older participants received $\$ 5$ for their participation. Twenty-six younger and 22 older adults participated in the first condition with no attended repetition trials, 23 younger and 22 older adults participated in the second condition with low levels of attended repetition trials, and 25 younger and 22 older adults participated in the third condition with high levels of attended repetition trials.

\section{Apparatus and Stimuli}

The apparatus and stimuli were similar to those in Simone, Carlisle, and McCormick (1998). The experiment was run using a Dell microcomputer to control stimulus presentation and to record RTs and error rates. The programming language was Microsoft Visual $\mathrm{C}++$. The computer was interfaced with an IBM 15-in. touch-sensitive color monitor (MicroTouch Systems, Inc.) located approximately $30 \mathrm{~cm}$ from the participant. Responses were made directly to the stimulus on this interactive touch screen monitor and were recorded by the computer. Response-sensitive areas overlapped the stimuli.

The stimuli were defined by their shape (plus sign, square, star, and triangle) and color (purple, green, yellow, and blue). Each stimulus was outlined in black and measured $3.8 \times 3.8 \mathrm{~cm}$. The four stimulus locations were underlined with a $4.5 \mathrm{~cm}$ black line, regardless of whether a stimulus was presented in the location or not. Although the total surface area of each stimulus was not identical (i.e., a square took up slightly more space than did a star), the response-sensitive area of the screen that responded to touch was equal for all the stimuli. The center stimulus measured $3.2 \times 3.2 \mathrm{~cm}$; it was not response sensitive. This center stimulus was white and denoted the shape of the target on the current trial. Peripheral stimuli included the target (with a shape identical to that of the center stimulus and filled with one of four colors) and a distractor (with a shape and color different from those of the target) in separate stimulus locations.

\section{Design}

Trials were presented in prime and probe pairs. Prime trials consisted of the center stimulus and a target and distractor in the periphery. There were four probe trials of interest (see Figure 1). A control probe target did not share location, shape, or color with the prime trial distractor or target. The attended repetition probe target shared location, shape, and color with the prime target (this trial type was not included in the $0 \%$ attended repetition trial condition). The ignored repetition probe target shared location, shape, and color with the prime distractor. In each of these probe trials, a distractor was also present. This probe distractor was not related to the prime trial or target in any way (location, shape, or color). The final trial type was used in the target-to-distractor condition. For this trial pair, the probe distractor shared the location, shape, and color of the prime target. The target in this probe was not related to the prime target or distractor in any way.

The proportion of attended repetition trials was varied in three conditions. The condition with no attended repetition trials $(0 \%)$ contained an equal number of control, ignored repetition, and target-todistractor trials. The condition with low levels of attended repetition trials $(25 \%)$ contained an equal number of attended repetition, control, ignored repetition, and target-to-distractor trial pairs. The condition with high levels of attended repetition trials (50\%) included three times as many attended repetition trial pairs but the same number of control, ignored repetition, and target-to-distractor trial pairs as in the other conditions. Total trial pair presentation across the three conditions varied, due to the inclusion of attended repetition trials in the $25 \%$ and $50 \%$ attended repetition trial conditions.

These trial pairs were presented in blocks of 27,36 , and 54 in the $0 \%, 25 \%$, and $50 \%$ attended repetition trial conditions, respectively. Each trial type was randomly presented 9 times within a block, except in the $50 \%$ attended repetition trials, where the attended repetition trial pairs were presented 27 times per block. Ordering within blocks was random. Control, ignored repetition, and target-todistractor trial pairs were presented 36 times, regardless of level of attended repetition trials. Attended repetition trials were presented 


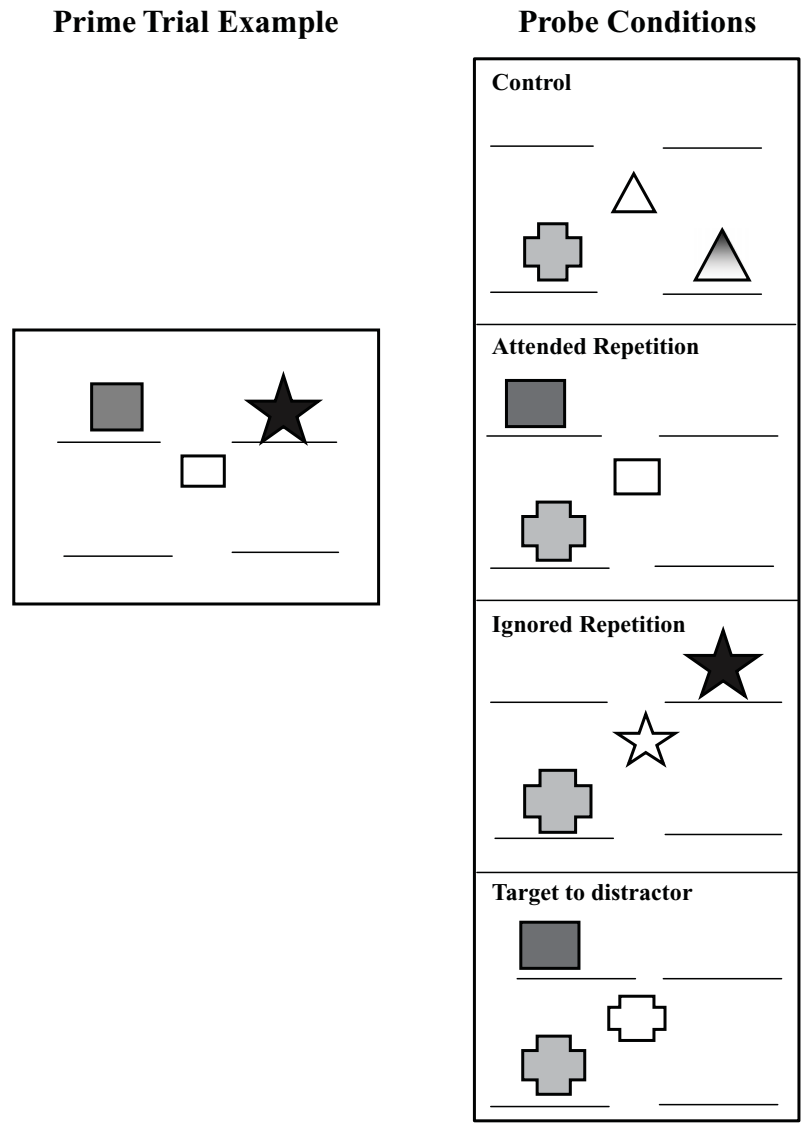

Figure 1. Examples of relevant probe conditions. Stimulus features included four locations, four shapes (star, square, triangle, and plus sign), and four colors (blue, green, yellow, and purple). In control trials, the prime and probe stimuli were different. In attended repetition trials, the prime and probe targets were identical. In ignored repetition trials, the prime distractor became the probe target. In target-to-distractor trials, the prime target became the probe distractor.

not at all in the $0 \%$ attended repetition trial condition, 36 times, in the $25 \%$ attended repetition trial condition, and 108 times in the $50 \%$ attended repetition trial condition.

\section{Procedure}

Each participant was randomly assigned to one of the three conditions. The participants were told that the smaller shape that would appear in the middle of the screen determined the target for that trial. Their task was to find the target in one of the four underlined peripheral locations and respond to it by touching the screen. On all the trials, a distractor stimulus (different shape and color than the target) appeared in one of the remaining three locations. The participants were instructed to respond to the target as quickly and accurately as possible. Incorrect responses made to either a distractor location or a blank stimulus location were signaled by a beep from the computer.

The participants were tested individually and were instructed to place their elbows on a pad in front of the monitor and to position their hands near the screen. The participants were encouraged to respond with both hands, using their right index finger to respond to the top right stimulus location and their right thumb to respond to the bottom right stimulus location. Their left index finger and thumb were used to respond to the top left and bottom left locations, respectively. However, if the participant was more comfortable using one hand or alternate hands, this was allowed, so each participant adopted a strategy that worked best for him or her. Whatever the strategy, the participants kept their hands near the surface of the screen and, if using two hands but two fingers, they simply moved their fingers slightly up or slightly down to reach the target. The participants were tested individually, and an experimenter remained in the room to ensure that the participants remained engaged in the task.

The trial pair sequence was as follows. A fixation cross appeared in the center of the screen for $500 \mathrm{msec}$. This fixation cross signaled that the trial was about to begin and that the participant should focus his or her attention to the center of the screen. Once the fixation cross disappeared, the stimulus display appeared immediately and remained illuminated until the participant made a response. This first trial was the prime. Once a response had been made to the prime trial, the screen went blank for $300 \mathrm{msec}$, followed by a $500-\mathrm{msec}$ fixation cross in the center of the screen. The probe trial, consisting of a center stimulus, a peripheral target, and a peripheral distractor, was then immediately presented. This display remained illuminated until a response was made. A response to the probe trial resulted in a blank screen for $500 \mathrm{msec}$.

The participants were given a brief practice session of 16 trials. This session was followed by a testing session of 216 trials, presented in 108 trial pairs (prime and probe) and in blocks of 54 trials in the $0 \%$ attended repetition trial condition. A total of 288 trials (144 trial pairs) were presented in blocks of 64 in the $25 \%$ attended repetition trial condition, and 432 total trials (216 trial pairs) were presented in blocks of 108 trials in the $50 \%$ attended repetition trial condition. The participants were allowed to rest between blocks, at which time they were given feedback about their average RT and number of errors in the previous block of trials.

\section{RESULTS}

\section{Treatment of Data}

Errors were recorded if the participant responded to a stimulus location that did not contain the target. All the trials were used to compute error rates for each participant. In calculation of the median RTs, error trials and trials immediately following an error were omitted, because of the variability that they introduced (Rabbitt, 1966). This might result in different sample sizes for each condition, which could cause a bias when median RTs are reported. However, Miller (1988) has shown that this bias is minimal when sample sizes are 20 or more. Means of median RTs and percentages of errors for all probe conditions (control, attended repetition, ignored repetition, and target to distractor) are shown in Table 1.

\section{Repetition Priming Probe Condition}

To determine whether the participants were faster on attended repetition trials than on control trials, and depending on the proportion of attended repetition trials, a 2 (age) $\times 2$ (attended repetition trial condition: $25 \%$ vs. $50 \%) \times 2$ (probe types: control vs. ignored repetition) mixed ANOVA was conducted, with age and attended repetition trial condition as between-subjects factors. There was a main effect of age on RTs $[F(1,88)=$ 115.67, $\left.M S_{\mathrm{e}}=89,664, p<.001\right]$, due to the slower overall response rates of the older adults. The main effect of attended repetition trial condition ( $25 \%$ vs. $50 \%)$ approached significance $\left[F(1,88)=3.75, M S_{\mathrm{e}}=89,664\right.$, 
Table 1

\begin{tabular}{|c|c|c|c|c|c|c|}
\hline \multicolumn{7}{|c|}{$\begin{array}{c}\text { Means of Median Response Times and Percentages of } \\
\text { Error for Younger and Older Adults }\end{array}$} \\
\hline \multirow[b]{2}{*}{ Condition Priming } & \multicolumn{3}{|c|}{ Younger Adults } & \multicolumn{3}{|c|}{ Older Adults } \\
\hline & RT & $\%$ Errors & Effect Size & RT & $\%$ Errors & Effect Size \\
\hline \multicolumn{7}{|l|}{$0 \%$ AR Trials } \\
\hline Control & 817 & 0.2 & & 1,146 & 0.7 & \\
\hline Ignored repetition & 835 & 0.7 & $-18^{*}$ & 1,188 & 0.8 & $-42^{*}$ \\
\hline Target to distractor & 797 & 0.5 & $20^{*}$ & 1,100 & 0.2 & $46^{*}$ \\
\hline \multicolumn{7}{|l|}{$25 \%$ AR Trials } \\
\hline Control & 730 & 0.3 & & 1,294 & 0.2 & \\
\hline Attended repetition & 684 & 0.1 & $46^{*}$ & 1,267 & 0.3 & $27^{*}$ \\
\hline Ignored repetition & 757 & 0.8 & $-27^{*}$ & 1,322 & 0.3 & $-28^{*}$ \\
\hline Target to distractor & 748 & 1.1 & $-18^{*}$ & 1,260 & 0.3 & $34^{*}$ \\
\hline \multicolumn{7}{|l|}{$50 \%$ AR Trials } \\
\hline Control & 755 & 0.7 & & 1,138 & 0.3 & \\
\hline Attended repetition & 684 & 0.2 & $71^{*}$ & 1,056 & 0.1 & $82^{*}$ \\
\hline Ignored repetition & 781 & 0.3 & $-26^{*}$ & 1,180 & 0.5 & $-42^{*}$ \\
\hline Target to distractor & 779 & 0.3 & $-24^{*}$ & 1,136 & 1.2 & 2 \\
\hline
\end{tabular}

Note-AR, attended repetition. Effect size equals control condition minus priming condition. ${ }^{*} p<.01$.

$p=.056]$, and the interaction between age and attended repetition trial condition was significant $[F(1,88)=4.91$, $\left.M S_{\mathrm{e}}=89,664, p<.05\right]$, suggesting that the responses of the older adults were somewhat faster with the highest proportion of attended repetition trials. The withinsubjects analysis showed that there was a main effect of probe types on RTs $\left[F(1,88)=62.61, M S_{\mathrm{e}}=2,326, p<\right.$ $.001]$, which interacted with proportion of attended repetition trials $\left[F(1,88)=7.67, M S_{\mathrm{e}}=2,326, p<.01\right]$ but did not interact with age $(F<1)$; the three-way interaction was not significant $\left[F(1,88)=1.15, M S_{\mathrm{e}}=2,326\right.$, $p>$.2]. Younger and older adults were faster on attended repetition trials than on control trials and were even faster in the $50 \%$ attended repetition trial condition. There was a main effect of probe types on errors $[F(1,88)=4.90$, $\left.M S_{\mathrm{e}}=0.47, p<.05\right]$, but no other main effects or interactions were significant. Fewer errors were made in attended repetition trials than in control trials.

To account for the longer RTs of the older adults, effect size was calculated (control minus attended repetition trials), and effect size data were submitted to a 2 (age) $\times$ 2 (attended repetition condition) ANOVA, with age and attended repetition trial condition $(25 \%$ and $50 \%)$ as the between-subjects factors. There was a main effect of attended repetition trial condition on RTs $[F(1,88)=7.67$, $\left.M S_{\mathrm{e}}=4,653, p<.01\right]$. Priming effect size was significantly larger in the $50 \%$ attended repetition trial condition. As can be seen from Table 1, as compared with control trials, both young and older adults were faster on attended repetition trials in the $25 \%$ attended repetition trial condition (younger, $46 \mathrm{msec}$; older, $27 \mathrm{msec}$ ) and were even faster in the high condition (younger, $71 \mathrm{msec}$; older, $82 \mathrm{msec})$. There was no effect of age $[F(1,88)=0.074$, $p>.7]$, and the interaction was not significant $[F(1,88)=$ $1.15, p>.2]$.

To summarize the findings, both younger and older adults were faster on trials in which the target was repeated than on control trials. This benefit in RT to target repetition was greatest when the proportion of attended repetition trials was greatest.

\section{Negative Priming and Target-to-Distractor Probe Conditions}

The effect of variable proportion of attended repetition trials on control, ignored repetition, and target-todistractor trials was examined in a 2 (age) $\times 3$ (attended repetition trial condition) $\times 3$ (probe type) repeated measures ANOVA. Age (younger and older adults) and attended repetition trial condition $(0 \%, 25 \%$, or $50 \%)$ were between-subjects factors. The within-subjects probe types were control, ignored repetition, and target-to-distractor trials. There was a main effect of age on RTs $[F(1,134)=$ $168.06, M S_{\mathrm{e}}=108,910, p<.001$ ], showing that, in general, the older adults were slower to respond than the younger adults. Varying the proportion of attended repetition trials in the three conditions did not have an effect on RTs $\left[F(2,134)=1.07, M S_{\mathrm{e}}=108,910, p>.3\right]$. The attended repetition trial condition $\times$ age interaction was significant $\left[F(2,134)=4.12, M S_{\mathrm{e}}=108,910, p<.05\right]$, suggesting that the RTs of the participants varied differently across the levels of attended repetition trials $(0 \%$, $25 \%$, and $50 \%$ ). For example, the RTs of the younger adults were shortest in the $25 \%$ attended repetition trial condition, whereas the group of older adults in the $25 \%$ attended repetition trial condition were, on average, slower to respond than were the older adults in the other two conditions.

There was a significant effect of probe type on response times $\left[F(2,268)=27.51, M S_{\mathrm{e}}=2,249, p<.001\right]$. Probe type interacted with age $\left[F(2,268)=9.51, M S_{\mathrm{e}}=2,249\right.$, $p<.001]$ and also with attended repetition trial condition $\left[F(4,268)=3.20, M S_{\mathrm{e}}=2,249, p<.05\right]$, but the threeway interaction was not significant $(F<1)$. As can be seen in Table 1, the young and older adults showed negative priming in all three conditions with varying proportions of attended repetition trials. Target-to-distractor effects 
varied as the participants were either faster or slower than on control trials, depending on the proportion of attended repetition trials. Specifically, the target-to-distractor effect was more positive (faster responses on target-to-distractor trials than on control trials) for the older adults across the different proportions of attended repetition trials.

There were no significant effects of errors on the between-subjects factors of age $(F<1)$ and attended repetition trial condition $\left[F(2,134)=2.58, M S_{\mathrm{e}}=1, p>.7\right]$, nor was the interaction significant $(F<1)$. Probe type also did not have an effect on errors $[F(2,268)=1.88$, $\left.M S_{\mathrm{e}}=1, p>.3\right]$. The probe type $\times$ attended repetition trial condition was the only significant interaction $\left[F(4,268)=3.13, M S_{\mathrm{e}}=1, p<.05\right]$, since the fewest errors were made in the condition with $50 \%$ attended repetition trials.

To ensure that the age effects were not due primarily to the slower responses made by the older adults, a further analysis used priming effect size as the dependent measure, which was calculated by subtracting control trial RTs from ignored repetition and target-to-distractor trial RTs. The analysis was a 2 (age) $\times 3$ (attended repetition trial condition: $0 \%, 25 \%$, or $50 \%) \times 2$ (priming effects: negative priming or target to distractor) repeated measures ANOVA. There was a main effect of attended repetition trial condition on effect size $[F(2,134)=3.05$, $\left.M S_{\mathrm{e}}=4,504, p=.05\right]$. As can be seen in Table 1, targetto-distractor effects changed with the addition of attended repetition trials. Importantly however, age did not influence effect size $[F(1,134)=1.58, p>.2]$, and the age $\times$ attended repetition trial condition interaction was not significant $[F(2,134)=0.91, p>$.4]. Therefore, the effects of age on responding are due to the priming effects of the age groups, not to overall slowing of older adults.

The within-subjects priming effects of negative priming and target-to-distractor priming were significant $\left[F(1,134)=38.06, M S_{\mathrm{e}}=2,998.27, p<.001\right]$. In addition, priming effects interacted both with age $[F(1,134)=$ $\left.13.48, M S_{\mathrm{e}}=2,998, p<.001\right]$ and with proportion of attended repetition trials $\left[F(2,134)=33.27, M S_{\mathrm{e}}=2,998\right.$, $p<.05]$. The three-way interaction was not significant $[F(2,134)=0.06, p>.9]$.

In post hoc Tukey HSD tests, pairwise comparisons were examined to determine whether RTs to ignored repetition and target-to-distractor trials were significantly different from RTs to control trials. All the effects were significant $(p<.01)$. Younger and older adults showed significant negative priming (slower RTs than in control trials) in all three attended repetition trial conditions $(0 \%$, $25 \%$, and $50 \%$ ). The younger and older adults were significantly faster on target-to-distractor trials than on control trials in the $0 \%$ attended repetition trials condition (20 and $46 \mathrm{msec}$, respectively). The younger adults were significantly slower on target-to-distractor trials in conditions with $25 \%$ and $50 \%$ attended repetition trials $(-18$ and $-24 \mathrm{msec}$, respectively). The older adults were significantly faster on target-to-distractor trials than on control trials in the condition with $25 \%$ attended repetition trials $(34 \mathrm{msec})$. There was no effect of target-to-distractor trials on the RTs of the older adults in the condition with $50 \%$ attended repetition trials $(2 \mathrm{msec})$.

To summarize the findings, the presence of attended repetition trials did not have an effect on negative priming in either younger or older adults, but attended repetition trials did have an effect on target-to-distractor responding, which was different in younger and older adults. Attended repetition trials slowed the responding of young adults on target-to-distractor trials and facilitated or had no effect on the responding of older adults on target-to-distractor trials.

\section{DISCUSSION}

Negative priming may result from distractor suppression of the ignored distractor during the processing of the prime target stimulus (e.g., Tipper, 1985). Or it may result from memory retrieval that occurs during the presentation of the probe display (e.g., Neill et al., 1992). Or negative priming may result from a bias to detect novelty (Christie $\&$ Klein, 2001). Some researchers have suggested that manipulations of target repetitions may alter the mechanism responsible for negative priming (e.g., May et al., 1995), and each theory makes different predictions about the influence of attended repetition trials on repetition priming, negative priming, and target-to-distractor trials.

The pattern of results presented here supports the findings of other researchers (e.g., Milliken et al., 2000) that an attentional bias for novelty, which is flexible and can be overridden, may be the primary mechanism responsible for priming effects in spatial localization tasks. Our findings further suggest that this novelty bias affects target and distractor processing separately and that, unlike in identitybased tasks (Kane et al., 1997), manipulating the processing of one (i.e., the target) does not affect processing of the other (i.e., the distractor). Our results also show that overriding the novelty bias for target processing is more selective in older than in younger adults and that older adults rely on the same inhibitory mechanism as younger adults for processing of the distractor.

\section{Target Processing: Repetition and Target-to-Distractor Priming}

Younger and older adults were faster to respond to repeating targets than in control trials, showing the repetitionpriming effect. In addition, repetition priming was more pronounced in both age groups when there was a high proportion of attended repetition trials. This finding is predicted by and may best be explained by the memory retrieval theory (e.g., Kane et al., 1997). However, this finding is not inconsistent with the flexible novelty bias mechanism proposed by Milliken et al. (2000). First, the high probability of repeated targets may have caused the novelty bias to be overridden. Second, repetition of the features of color and identity in conjunction with repetition of location may also have obscured the bias to avoid recently selected locations. Since the level of repetition priming increased as the proportion of attended repetition trials added to the condition increased, evidence points to 
memory retrieval of the target as the mechanism responsible for obscuring the novelty bias.

Target-to-distractor processing was different between the age groups, with the exception of responding in the $0 \%$ attended repetition trial condition, in which both younger and older adults were faster on target-to-distractor trials than on control trials. This facilitation in responding was predicted by the novelty bias mechanism. Because the target location had already been inspected in the prime trial, there was a bias against returning attention to that location in the probe trial. Therefore, the current target was selected more quickly.

Younger adults were slower to respond on the targetto-distractor trials than on control trials in the conditions with variable proportions of attended repetition trials $(25 \%$ and $50 \%$ ). Although the novelty bias mechanism predicts faster responding when a target becomes a distractor, the presence of attended repetition trials has overridden the novelty bias and resulted in a slowdown on target-todistractor trials. The presence of repeated targets encouraged participants to adopt the strategy of memory retrieval for all responding, a strategy that is beneficial when the target is repeated as a target but causes a disadvantage in RT if the target is now a distractor.

Overall, older adults showed a more positive effect on target-to-distractor trials (faster responses, as compared with control trials) than did younger adults, as is predicted by the theory of a novelty bias mechanism of attention. Older adults showed facilitation to the target-to-distractor probe in the $25 \%$ attended repetition trial condition, and there was no effect on responding in the $50 \%$ attended repetition trial condition, whereas younger adults were slower in both conditions, due to memory retrieval. Therefore, although the presence of attended repetition trials caused memory retrieval to obscure the novelty bias, leading to repetition priming in older adults, the novelty bias mechanism was not obscured whenever a target was repeated, but only when a target was repeated as a target. Therefore, obscuring of the novelty bias was selective, and memory retrieval from target processing did not influence targetto-distractor responding in older adults.

\section{Distractor Processing: Negative Priming}

We propose that memory retrieval was responsible for overriding the novelty bias in target processing. Because the presence of attended repetition trials did not affect the negative priming observed in the younger or older adults in this spatial localization task, we conclude that target processing is separate from distractor processing, a finding that is contrary to that of Kane and colleagues (Kane et al., 1994; Kane et al., 1997), who found that the addition of attended repetition trials influenced negative priming.

Methodological differences between the present study and those of Kane and colleagues may have been responsible for the discrepant findings. Whereas Kane et al. (1997) required participants to verbally identify the target, we used a spatial localization task that involved locating a target and distractor in specific spatial locations. The bias to search novel locations in location-based tasks may affect the processing of targets and distractors (e.g., Connelly \& Hasher, 1993), enabling the processing of each to be independent and, perhaps, involving different mechanisms.

It is difficult to differentiate between an inhibition of return that is due to a novelty bias and a negative priming that is due to suppression of distracting information in location-based tasks, and we were unable to do so in this experiment. Nonetheless, the processes may be related. Although Christie and Klein (2001) have suggested that ignoring a distractor is not necessary in location-based tasks and, therefore, negative priming in location-based tasks is due to inhibition of return, Milliken et al. (2000) and Buckolz et al. (2002) have argued that inhibition of return effects and location-based negative priming effects may share a common inhibition mechanism.

Because novelty bias can be overridden in target processing, it may be possible to differentiate between distractor suppression and novelty bias explanations of negative priming if it can be demonstrated that the novelty bias in distractor processing can also be overridden. It might be possible to eliminate negative priming by obscuring the novelty bias in an experiment with variable proportions of ignored repetition trials. To our knowledge, this experiment has not been conducted. Buckolz et al. (2002) were able to reduce but not eliminate the negative priming effect by using a $100 \%$ valid cue to the target location. Therefore, with advance knowledge of where the target would be located and no need to inspect the location of the distractor, negative priming was still found. Buckolz et al. concluded that inhibition of return and negative priming utilized a shared inhibitory mechanism.

To summarize our findings and conclusions, we found that a novelty bias mechanism was responsible for target and distractor processing in both younger and older adults. The bias to search for novel locations was overridden by memory retrieval in both age groups when a benefit to return to a location was detected, such as when the target was repeated as a target (attended repetition trials). Older adults continued to use the novelty bias mechanism in target processing with a low proportion of attended repetition trials, whereas younger adults were less selective in overriding the novelty bias when the target became a distractor. Younger and older adults also showed negative priming that was unaffected by target processing, suggesting that target processing and distractor processing are separate in spatial localization tasks. In contrast to Kane et al. (1997), who found that older adults showed negative priming only when attended repetition trials encouraged memory retrieval, we found significant and comparable negative priming in younger and older adults that may best be explained by inhibitory processes underlying novelty bias and/or distractor suppression.

\section{REFERENCES}

Buckolz, E., Boulougouris, A., O’Donnell, C., \& Pratt, J. (2002). Disengaging the negative priming mechanism in location tasks. European Journal of Cognitive Psychology, 14, 207-225.

Christie, J., \& Klein, R. M. (2001). Negative priming for spatial location? Canadian Journal of Experimental Psychology, 55, 24-38. 
Connelly, S. L., \& Hasher, L. (1993). Aging and the inhibition of spatial location. Journal of Experimental Psychology: Human Perception \& Performance, 19, 1238-1250.

Fox, E. (1995). Negative priming from ignored distractors in visual selection: A review. Psychonomic Bulletin \& Review, 2, 145-173.

Gamboz, N., Russo, R., \& Fox, E. (2000). Target selection difficulty, negative priming, and aging. Psychology \& Aging, 15, 542-550.

Kane, M. J., Hasher, L., Stoltzfus, E. R., Zacks, R. T., \& Connelly, S. L. (1994). Inhibitory attentional mechanisms and aging. Psychology \& Aging, 9, 103-112.

Kane, M. J., May, C. P., Hasher, L., Rahhal, T., \& Stoltzfus, E. R. (1997). Dual mechanisms of negative priming. Journal of Experimental Psychology: Human Perception \& Performance, 23, 632-650.

May, C. P., Kane, J. M., \& Hasher, L. (1995). Determinants of negative priming. Psychological Bulletin, 118, 35-54.

McCrae, C., \& Abrams, R. (2001). Age-related differences in objectand location-based inhibition of return of attention. Psychology \& Aging, 16, 437-449.

McDowd, J. M., \& Oseas-Kreger, D. M. (1991). Aging, inhibitory processes, and negative priming. Journal of Gerontology, 46, P340-P345.

Miller, J. O. (1988). A warning about median reaction time. Journal of Experimental Psychology: Human Perception \& Performance, 14, 539-543.

Milliken, B., Tipper, S. P., Houghton, G., \& Lupiáñez, J. (2000). Attending, ignoring, and repetition: On the relation between negative priming and inhibition of return. Perception \& Psychophysics, 62, 1280-1296.

Neill, W. T., Valdes, L. A., \& Terry, K. M. (1995). Selective attention and the inhibitory control of cognition. In F. M. Dempster \& C. J. Brainerd (Eds.), Interference and inhibition in cognition (pp. $207-$ 261). San Diego: Academic Press.

Neill, W. T., Valdes, L. A., Terry, K. M., \& Gorfein, D. S. (1992) The persistence of negative priming: II. Evidence for episodic trace retrieval. Journal of Experimental Psychology: Learning, Memory, \& Cognition, 18, 993-1000.
PARK, J., \& KANWISHER, N. (1994). Negative priming for spatial locations: Identity mismatching, not distractor inhibition. Journal of Experimental Psychology: Human Perception \& Performance, 20, 613-623.

Posner, M. I., \& Cohen, Y. (1984). Components of visual orienting. In H. Bouma \& D. G. Bouwhuis (Eds.), Attention and performance X: Control of language processes (pp. 531-556). Hillsdale, NJ: Erlbaum.

RABBITT, P. M. A. (1966). Errors and error correction in choice response tasks. Journal of Experimental Psychology, 71, 264-272.

Schooler, C., Neumann, E., Caplan, L. J., \& Roberts, B. (1997). Continued inhibitory capacity throughout adulthood: Conceptual negative priming in younger and older adults. Psychology \& Aging, 12, 667-674.

Simone, P. M., \& Baylis, G. C. (1997). Selective attention in a reaching task: The effect of normal aging and Alzheimer's disease. Journal of Experimental Psychology: Human Perception \& Performance, 23, 595-608.

Simone, P. M., Carlisle, E. A., \& McCormick, E. B. (1998). Effect of defining features on inhibition is a spatial localization task. Journal of Experimental Psychology: Human Perception \& Performance, 24 993-1003.

Simone, P. M., \& McCormick, E. B. (1999). Effect of a defining feature on negative priming across the life span. Visual Cognition, 6 , 587-606.

Sullivan, M. P., \& Faust, M. E. (1993). Evidence for identity inhibition during selective attention in old adults. Psychology \& Aging, 8, 589-598.

Sullivan, M. P., Faust, M. E., \& Balota, D. A. (1995). Identity negative priming in older adults and individuals with dementia of the Alzheimer type. Neuropsychology, 9, 537-555.

TIPPER, S. P. (1985). The negative priming effect: Inhibitory priming by ignored objects. Quarterly Journal of Experimental Psychology, 37A, 571-590.

(Manuscript received July 30, 2003; revision accepted for publication January 16, 2005.) 